\title{
Predicting Cervical Cancer Cases Resulting in Biopsies Using Machine Learning Techniques
}

\author{
Tenali Pranuthi
}

Lovely Professional University, Punjab, India

\begin{abstract}
Article Info

Volume 7, Issue 6

Page Number: 28-37

Publication Issue :

November-December-2021

\section{Article History}

Accepted : 09 Nov 2021

Published : 19 Nov 2021

There are various algorithms and methodologies used for automated screening of cervical cancer by segmenting and classifying cervical cancer cells into different categories. This study presents a critical review of different research papers published that integrated ML methods in screening cervical cancer via different approaches analyzed in terms of typical metrics like dataset size, drawbacks, accuracy etc. An attempt has been made to furnish the reader with an insight of Machine Learning algorithms like SVM (Support Vector Machines), k-NN (kNearest Neighbors), RFT (Random Forest Trees), for feature extraction and classification. This paper also covers the publicly available datasets related to cervical cancer. It presents a holistic review on the computational methods that have evolved over the period of time, in detection of malignant cells.

In this paper, we are going to train our model using various machine learning techniques and all the models thus made are compared in terms of accuracy, precision and recall.
\end{abstract}

Keywords : Ensembling, Supervised learning, unsupervised learning, Random Forest, Decision trees, cancer, biopsy.

\section{INTRODUCTION}

Cervical cancer is a malignant tumour starting in the cells of a woman's cervix, and possibly spreading or metastasizing to other parts of her body. The cervix is part of a woman's reproductive system, located below the uterus. In most cervical cancer cases, the tumours develop from precancerous changes in the cervix, and can take several years to develop.
About 13,800 new cases of invasive cervical cancer will be diagnosed. About 4,290 women will die from cervical cancer. In the detection of cervical cancer, machine learning techniques have been of much help contributing to the medical stream.

In the paper titled New Features of Cervical Cells for Cervical Cancer Diagnostic System Using Neural Network by Mustafa et al [3], it has been stated that though Pap test is the most popular and effective test 
for cervical cancer, Pap test does not always produce good diagnostic performance. In the paper Preprocessing for Automating Early Detection of Cervical Cancer, by Debasis Bhattacharyya et al.[4] states that In Cervigram, cervix region occupies about half of the raw cervigram image. Other parts of the image contain inconsequential information. This irrelevant information can muddle automatic identification of the tissues within the cervix. Asselin et al.[5] discuss the imaging methods available to provide appropriate biomarkers of tumor structure and function using selective regions of interest (ROI), Cluster analysis and Histogram analysis. Turid Torheim et.al[6] present the paper with texture analysis methods and classification by using SVM to identify the cured and relapsed images. S. Jagadeeswari and S. Malarkhodi[7] presented a paper on classification by using an Artificial Neural Network to identify the normal and abnormal tumor images with Fourier transform and Gaussian low pass filter. Rupinderpal and Rajneet presented a noise removal method using discrete wavelet transform.

Here, we have tried to detect the cancer using ML techniques along with ensembling.

\section{Literature Review}

Supervised Learning Techniques:

Supervised learning is the machine learning task of learning a function that maps an input to an output based on example input-output pairs. It infers a function from labeled training data consisting of a set of training examples.

The supervised machine learning techniques that we used here are Logistic Regression, Random Forest Classifier, SVM.

\section{Random Forest:}

Random Forest is an ensemble algorithm which creates many decision trees (a forest), and applies them to multiple subsets of the dataset, creating multiple classification results. The Random Forest
Classifier uses a voting system to make its final classification prediction, with each tree voting, and chooses the class with the most votes. An alternative voting measure is using weights to assign the impact of a decision tree's result, with trees with high errors getting low weightings, and vice versa. In this voting system, trees with low error rates have a higher impact on the final classification decision.

\section{Random Forest Simplified}

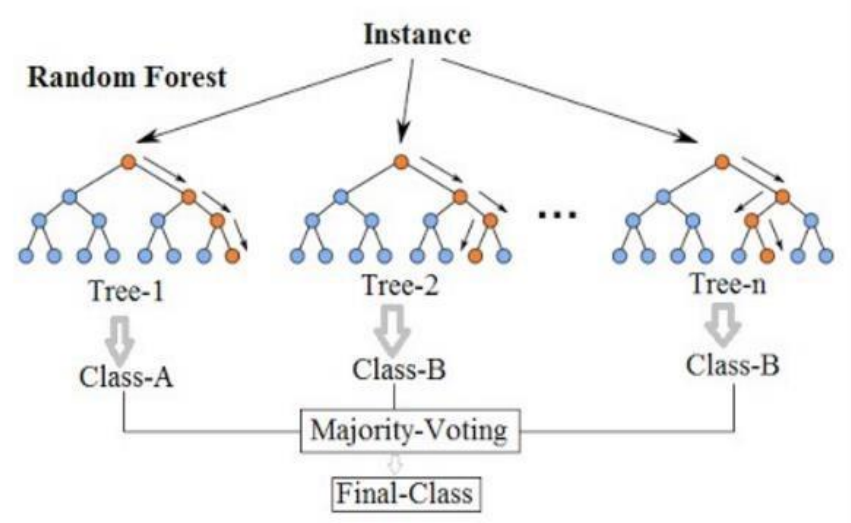

The Random Forest Classifier splits the dataset into a training set and testing set by sampling with replacement, until approximately one-third of the data is remaining, which is used for testing the classifier. Before applying the classifier to the data, you must determine how many trees each forest should contain, and the minimum number of nodes required in order for the tree to split. Advantages:

1.It's works well with noisy data and it reduces overfitting. Since the end result is an average or majority vote of multiple classification results, the classifier has a significantly lower chance of overfitting the data.

2.Since there are multiple forests, not every forest is necessarily affected by noisy data.

Disadvantages:

1.They are much more complex than normal decision trees, thus are harder to understand and visualize. 
2.Because there are many more trees being created and used than in a normal decision tree, it is more computationally expensive.

\section{Support Vector Machine (SVM):}

A Support Vector Machine is a classification technique which attempts to separate the different classes of data by finding a decision boundary which maximizes the margin. The SVM represents this boundary by using support vectors. Although there are infinitely many different hyperplanes that could be selected to separate the data, the hyperplane with the largest margin often performs better, as it leaves more room for any perturbations to the decision boundary without having an impact on the classification.

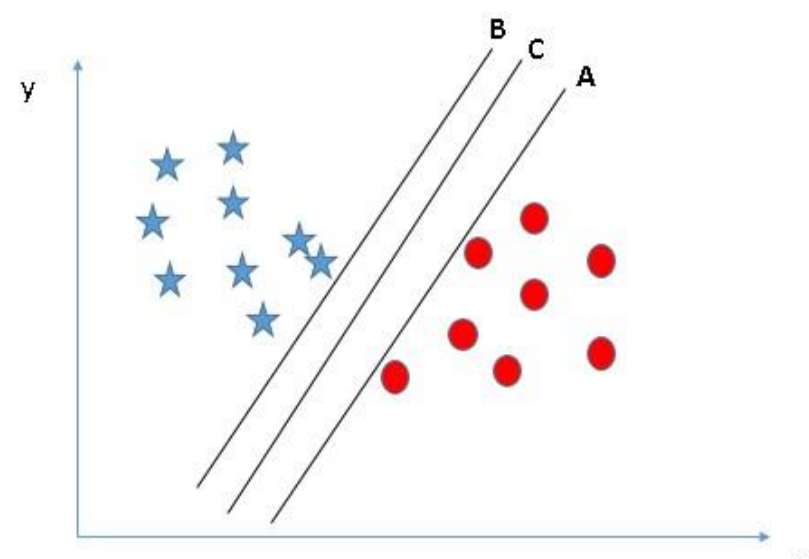

Advantages:

- They work well with high-dimensional data, avoid the curse of dimensionality, and they still work well in cases where there are more dimensions than there are samples of data.

Disadvantage:

- They are harder to analyze, as they do not give out a probability score.

Logistic Regression:
This is a learning technique employed when the output of training data is in the form of groups called classes.

In our model, the output is 0 if the patient is negative with cancer and 1 if she is diagnosed positive.

Advantages of Logistic Regression:

- Logistic Regression performs well when the dataset is linearly separable.

- Logistic regression is less prone to over-fitting but it can overfit in high dimensional datasets. You should consider Regularization (L1 and L2) techniques to avoid over-fitting in these scenarios.

Disadvantages:

- Main limitation of Logistic Regression is the assumption of linearity between the dependent variable and the independent variables. In the real world, the data is rarely linearly separable. Most of the time data would be a jumbled mess.

- Logistic Regression can only be used to predict discrete functions. Therefore, the dependent variable of Logistic Regression is restricted to the discrete number set. This restriction itself is problematic, as it is prohibitive to the prediction of continuous data.

The unsupervised technique used is $\mathrm{K}$ means clustering.

K-Means Clustering:

It is an unsupervised approach to classifying data which tries to make clusters of similar data. Each data point is compared to randomly selected centroids, and placed in the neighborhood of its nearest cluster (using Euclidean distance). The number of clusters must be defined at the beginning of the model. After selecting the initial centroids, the distances are computed and the data is assigned to centroid, and the centroids are recomputed multiple times until they don't move around anymore. 
$\mathrm{K}$-means clustering performs well and is easy to understand the visualization of the data, However, $\mathrm{k}$ means clustering doesn't perform well when the data is of different sizes or densities, and has problems when the data contains outliers.

\section{Advantages:}

- Relatively simple to implement.

- Scales to large data sets.

- Guarantees convergence.

Disadvantages:

- Choosing k manually.

- Being dependent on initial values.

\section{About the dataset:}

There are 36 attributes in the dataset, consisting of 32 risk factors, and 4 target variables (the last four attributes):

The dataset, "Cervical Cancer Risk Factors for Biopsy" was obtained from

\begin{tabular}{|c|c|c|}
\hline Name & Type & Description \\
\hline Age & Integer & Age of patient \\
\hline Number of sexual partners & Integer & Total number of previous sexual partners \\
\hline First sexual intercourse (age) & Integer & Age at which the patient first had sexual intercourse \\
\hline Num of pregnancies & Integer & Total number of previous pregnancies \\
\hline Smokes & Boolean & Yes/No \\
\hline Smokes (years) & Real & Number of years they smoked \\
\hline Smokes (packs/year) & Real & Number of packs they smoke per year \\
\hline Hormonal contraceptives & Boolean & Yes/No: Have they used hormonal contraceptives \\
\hline Hormonal contraceptives (years) & Real & Number of years they used hormonal contraceptives \\
\hline IUD & Boolean & $\begin{array}{l}\text { Yes/No: Have they had an IUD (form of hormonal } \\
\text { contraceptive) }\end{array}$ \\
\hline IUD (years) & Real & Number of years they used an IUD \\
\hline STD & Boolean & $\begin{array}{l}\text { Yes/No: Have they had an STD (Sexually Transmitted } \\
\text { Disease) }\end{array}$ \\
\hline STDs (number) & Integer & Number of STD's they've had \\
\hline STDS: condylomatosis & Boolean & Yes/No: Have they had condylomatosis \\
\hline STDs: cervical condylomatosis & Boolean & Yes/No: Have they had cervical condylomatosis \\
\hline STDs: vaginal condylomatosis & Boolean & Yes/No: Have they had vaginal condylomatosis \\
\hline $\begin{array}{l}\text { STDs: vulvo-perineal } \\
\text { condylomatosis }\end{array}$ & Boolean & Yes/No: Have they had vulvo-perineal condylomatosis \\
\hline STDs: syphilis & Boolean & Yes/No: Have they had syphilis \\
\hline STDs: pelvic inflammatory disease & Boolean & Yes/No: Have they had pelvic inflammatory disease \\
\hline STDs: genital herpes & Boolean & Yes/No: Have they had genital herpes \\
\hline STDs: molluscum contagiosum & Boolean & Yes/No: Have they had molluscum contagiosum \\
\hline STDs: AIDS & Boolean & Yes/No: Have they had AIDS \\
\hline STDS: HIV & Boolean & Yes/No: Have they had HIV \\
\hline STDS: Hepatitis B & Boolean & Yes/No: Have they had Hepatitis B \\
\hline STDS: HPV & Boolean & Yes/No: Have they had HPV \\
\hline STDs: Number of diagnosis & Integer & Number of diagnoses of STDS \\
\hline STDs: Time since first diagnosis & Integer & Years since first STD diagnosis \\
\hline STDs: Time since last diagnosis & Integer & Years since last STD diagnosis \\
\hline Dx: Cancer & Boolean & Yes/No: Have they had a dx test for cervical cancer \\
\hline Dx: CIN & Boolean & $\begin{array}{l}\text { Yes/No: Have they had a dx test for CIN (Cervical } \\
\text { Intraepithelial Neoplasia) }\end{array}$ \\
\hline Dx: HPV & Boolean & Yes/No: Have they had a dx test for HPV \\
\hline $\mathrm{Dx}$ & Boolean & Yes/No: Have they had a dx test \\
\hline Hinselmann: target variable & Boolean & Yes/No: Have they had a Hinselmann test (colonoscopy) \\
\hline Schiller: target variable & Boolean & $\begin{array}{l}\text { Yes/No: Have they had a Schiller test (using iodine to detect } \\
\text { cancer cells) }\end{array}$ \\
\hline Cytology: target variable & Boolean & Yes/No: Have they had a cytology-based test (Pap test) \\
\hline Biopsy: target variable & Boolean & Yes/No: Have they had a biopsy \\
\hline
\end{tabular}

the Kaggle. The dataset contains habits, demographic information, and medical history of 858 patients from the hospital.

\section{Proposed Methodology:}

Visualization of data:

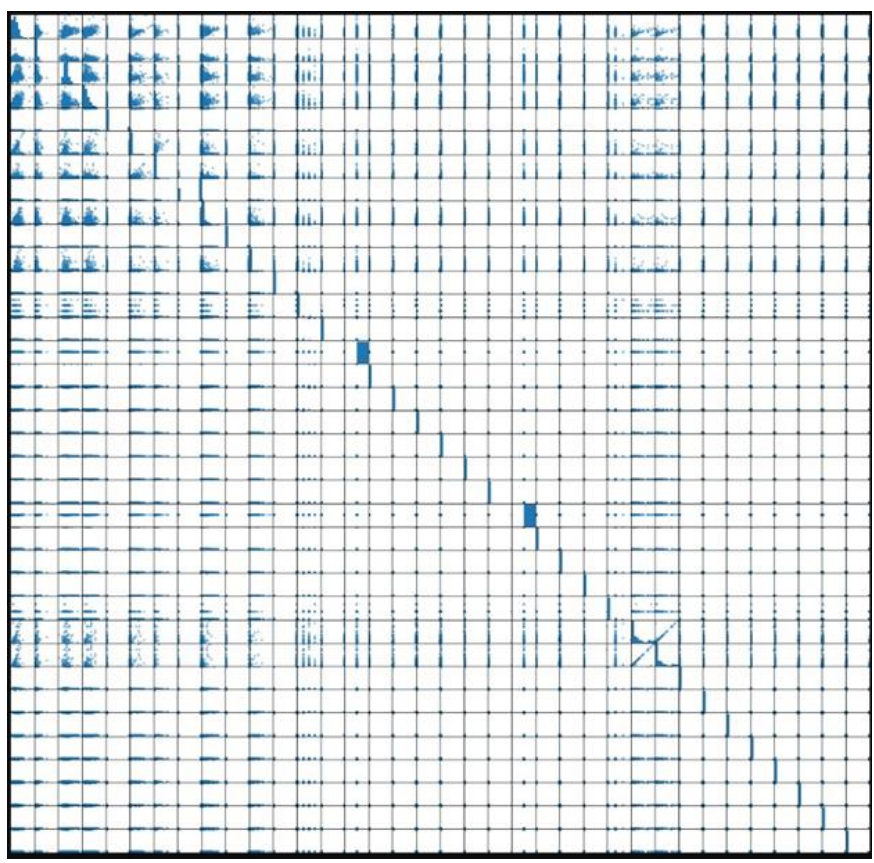

The above is the scattered matrix plot obtained on plotting the features of the dataset as a plot. The scatter matrix is

advantageous in revealing strong correlations between specific attributes, or showing that two attributes don't have

any sort of correlation. In looking at the "Biopsy" column (the furthest right), I could see many scatterplots that didn't have strong correlations, leading to the fact that there are some potential attributes that are redundant or irrelevant and could be removed from training.

Data Preprocessing:

Imputation: 
Because of the large number of missing values in this dataset, I decided to replace the missing values instead of eliminating them. This way I could be sure not to lose any important data, and try to make the most accurate estimate of the missing data.

The numerical values are replaced with the median of that particular column and the categorical values are replaced with the mode value.

Standardization \& Normalization:

Due to the fact that some of the attributes in the dataset are binary values and some are not, standardizing and normalizing the dataset is advantageous in evening out the values and helping to solve imbalance problems. Using a built-in function for standardizing/normalizing data in scikitlearn, the dataset now is standardized.

Also there are many attributes in the dataset which has weak correlations with the target and those are removed.

If two columns or attributes that are non- target values are having strong correlation, one of the attribute can be removed in order to reduce the dimensional complexity. Here Random Forest Classifier or PCA can be used to do dimensional reduction.

\begin{tabular}{rrr} 
& feature & rfc \\
\hline 0 & Age & 0.096112 \\
1 & Number of sexual partners & 0.033912 \\
2 & First sexual intercourse & 0.065913 \\
3 & Num of pregnancies & 0.061399 \\
4 & Smokes & 0.015895 \\
5 & Smokes (years) & 0.024683 \\
6 & Smokes (packs/year) & 0.020065 \\
7 & Hormonal Contraceptives & 0.016056 \\
8 & Hormonal Contraceptives (years) & 0.061133 \\
9 & IUD & 0.010110 \\
10 & IUD (years) & 0.016989 \\
11 & STDs & 0.003390 \\
12 & STDs (number) & 0.008450 \\
13 & STDs:condylomatosis & 0.001102 \\
14 & STDs:cervical condylomatosis & 0.000000 \\
\hline
\end{tabular}

Now as the data is ready to use to train the models, we are all set to proceed with training the models.

Decision Tree classifier: The criterion used here is gini index with parameters set to max_leaf_nodes $=$ None, min_samples_leaf $=14$, min_samples_split $=5$, random_state $=1$

Logistic Regression: The parameters are set to $C=4$, penalty=' 12 '.

And also set to $\mathrm{C}=4$, penalty='11' in another model.

KNN:

The parameters are set to $\mathrm{n} \_$neighbors $=5, \mathrm{p}=2$, metric $=$ 'minkowski'.

Random Forest Classifier:

The parameters are set to max_depth $=5$,

n_estimators $=10$, max_features $=1$,

SVM:

The kernel is set to "rbf" with $\mathrm{C}=100$,

gamma $=15 \mathrm{n} \_$jobs $=10$

\section{Results and Discussion:}

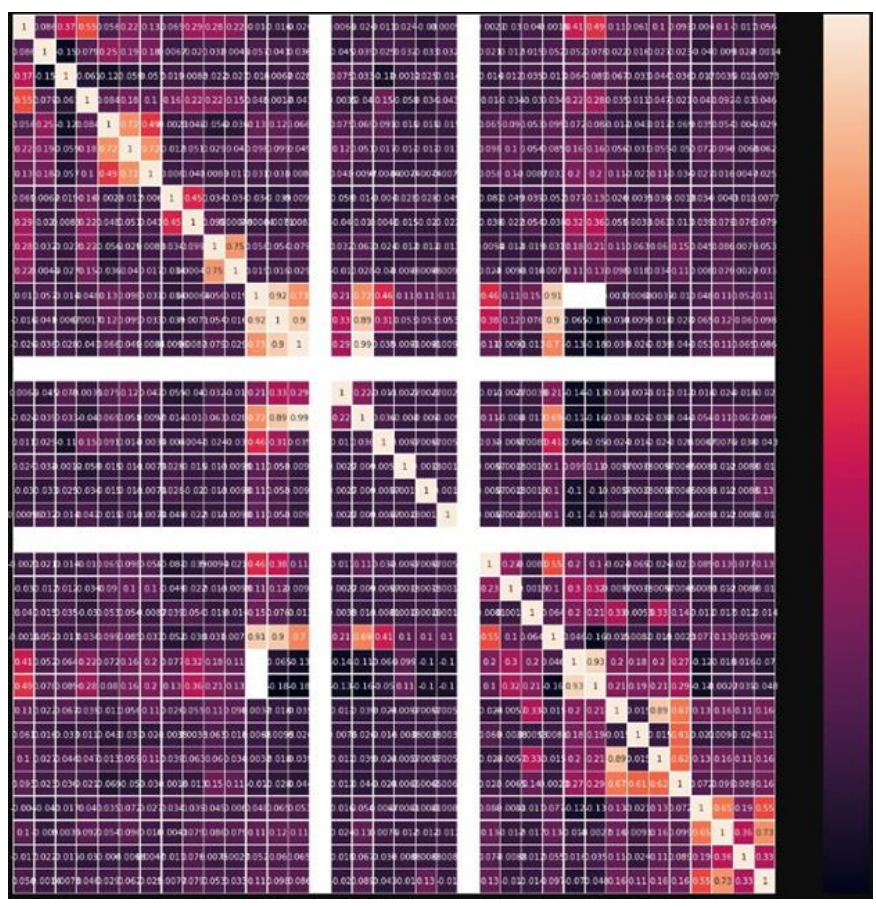

Correlation matrix of the features between datasets 


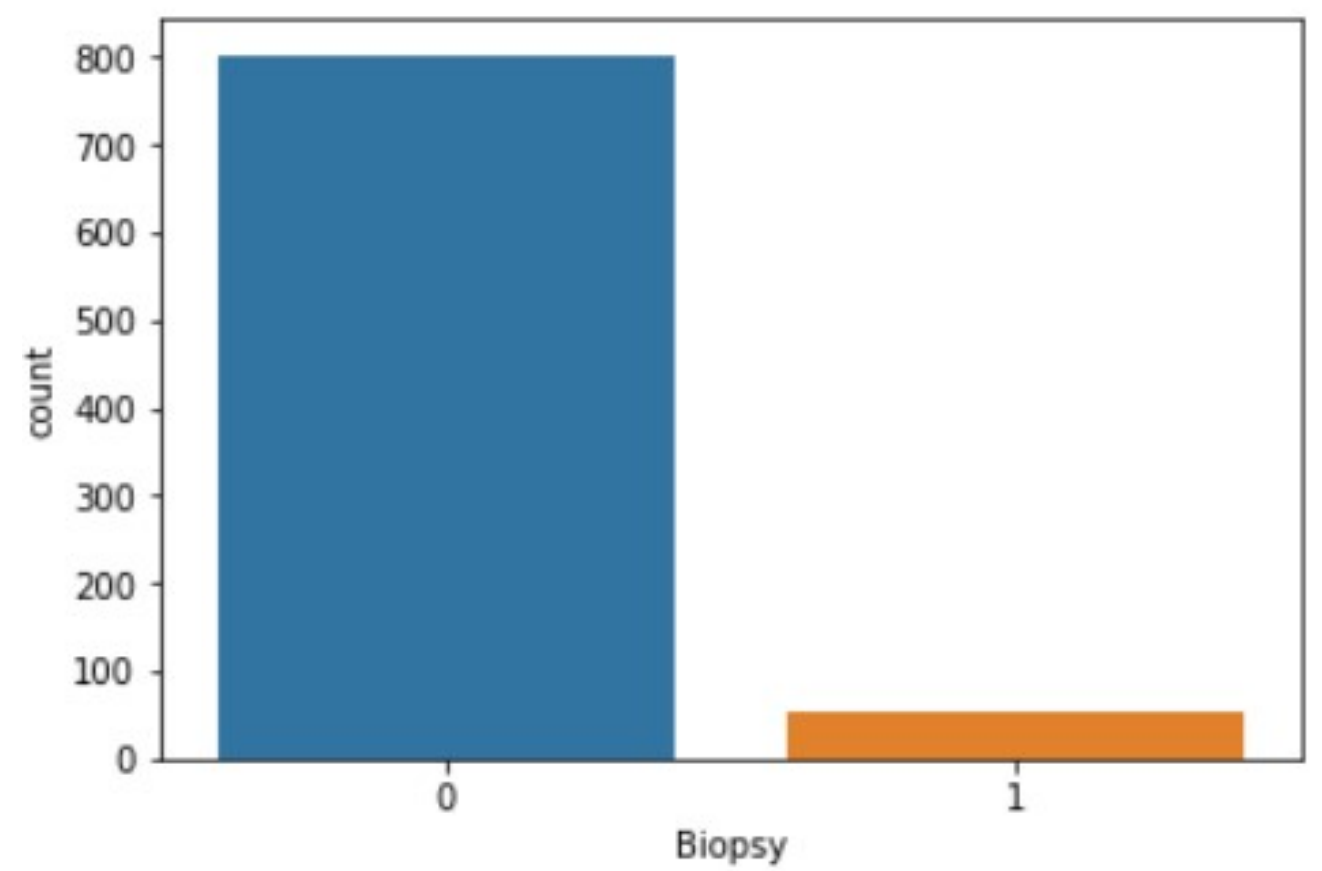

True positives in the data

Comparison of different models used in detecting cervical cancer:

\begin{tabular}{|c|c|c|c|c|c|c|}
\hline Model & Accuracy & Class & Precision & Recall & F1 score & Support \\
\hline \multirow[t]{2}{*}{ Decision Tree } & \multirow[b]{2}{*}{$95.38 \%$} & 0 & 0.97 & 1.0 & 0.97 & 242 \\
\hline & & 1 & 1.0 & 0.52 & 0.69 & 16 \\
\hline \multirow{2}{*}{$\begin{array}{l}\text { Logistic } \\
\text { Regression }\end{array}$} & \multirow[b]{2}{*}{$94.5 \%$} & 0 & 0.98 & 0.98 & 0.96 & 242 \\
\hline & & 1 & 0.73 & 0.52 & 0.61 & 16 \\
\hline \multirow[b]{2}{*}{ Knn } & \multirow[b]{2}{*}{$93.75 \%$} & 0 & 0.94 & 1.00 & 0.97 & 242 \\
\hline & & 1 & 0.01 & 0.00 & 0.03 & 16 \\
\hline \multirow[b]{2}{*}{ K means } & \multirow[b]{2}{*}{$76.27 \%$} & 0 & 0.93 & 0.23 & 0.37 & 242 \\
\hline & & 1 & 0.02 & 0.06 & 0.03 & 16 \\
\hline \multirow{2}{*}{$\begin{array}{l}\text { Random } \\
\text { Forest }\end{array}$} & \multirow[b]{2}{*}{$93.7 \%$} & 0 & 0.94 & 1.00 & 0.97 & 242 \\
\hline & & 1 & 0 & 0 & 0.2 & 16 \\
\hline \multirow[b]{2}{*}{ SVM } & \multirow[b]{2}{*}{$91 \%$} & 0 & 0.94 & 0.96 & 0.95 & 242 \\
\hline & & 1 & 0.1 & 0.06 & 0.08 & 16 \\
\hline \multirow[b]{2}{*}{ Perceptron } & \multirow[b]{2}{*}{$92.63 \%$} & 0 & 0.94 & 0.98 & 0.96 & 242 \\
\hline & & 1 & 0.2 & 0.06 & 0.1 & 16 \\
\hline
\end{tabular}

As far as the models are all trained and tested, the As the decision trees can avoid overfitting, we don't decision tree model has got the highest accuracy see the problem of overfitting in our model. when compared to all the other models. 
Among the very first works in cervical cancer detection stands the work by P. Mitra in the year 2000 where staging was done by amalgamation of ID3 and GAs, where GAs were mainly used for refining the architecture. In the work by J. Zhang et al in 2004 the use of SVMs was more desirable because they had small data set of 40 images containing 149 cells. Back in that time medical imaging data set was meagre and SVMs provide accurate result(clearer decision boundary) on small size batch. In paper by R. Vidya and G. M. Nasira [9], CART algorithm was initially implemented to check the feasibility of the task. On realizing pretty successful results, advanced algorithms RFT and RFT with k-NN were implemented leading up to an accuracy of $94.77 \%$.

The Decision Tree model that has been trained here produced an accuracy of $95.3 \%$. So, the decision tree model is said to be highly useful in case of cervical cancer detection using the cervical cancer risk classification dataset.

\section{Parameter tuning:}

- The logistic regression technique that is set with ' 12 ' penalty has more accuracy than that of '11'

- The decision tree and random forest has same accuracy because the parameters of both the models are set same.

- $\quad$ No matter if we take one tree or a forest.

- When the number of jobs are being increased the accuracy of random forest went down. So the optimum values is set to 10 .

- The accuracy of perceptron has no effect though the learning rate is changed.

\section{CONCLUSION}

In conjunction with more accurate diagnostics, AI has the potential to bring down the cost of unwanted interventions for cervical cancer screening. Early detection will promise a greater rate of patients prognosis especially in case of non-invasive cancer. The papers discussed above made use of independent data sources, consequently a base for comparing algorithms on a single scale was hard to define. Multiple algorithms have been applied for segmenting cell cytoplasm, nuclei and other cell components and classifying cells into different categories. In the view of the known stages of cancer, the accuracy of each algorithm, Decision Tree has proved to yield highest accuracy for classifying cervical cancer risk.

Although it can be difficult to compare the results of the supervised models with the unsupervised model, in this case, it is clear that the Decision Tree Classifier performed better than the other three models. Not only did it have a higher overall accuracy than the Random Forest and Support Vector Machine classifiers, but it also had the highest precision, accuracy, and F1 measures, signifying that it was better at correctly classifying the positive class. In comparing this to the unsupervised K-means clustering model, it's hard to find an exact difference in performance between the two, but the Gradient Boosting Classifier was able to clearly classify a large amount of the test set correctly, whereas the K-means cluster model struggled to separate the $\mathrm{y}$ values from the $\mathrm{X}$ values.

\section{REFERENCES}

[1]. S. Sharma, "Cervical Cancer stage prediction using Decision Tree approach of Machine Learning", International Journal of Advanced Research in Computer and Communication Engineering vol. 5, Issue 4, 2016. 
[2]. J. Zhang and Y. Liu, "Cervical Cancer Detection Using SVM Based Feature Screening”, 2004. [3] P. K. Malli, S. Nandyal, "Machine learning Technique for detection of Cervical Cancer using k-NN and Artificial Neural Network", International Journal of Emerging Trends \& Technology in Computer Science (IJETTCS), 2017

[3]. Cervical cancer (risk factor) data set UCI Machine Learning Repository, https://archive.ics.uci.edu/ml/datasets/Ce rvical+cancer+\%28Risk+Factors\%29

[4]. https://www.stat.berkeley.edu/ breiman/ RandomForests/cc_home.html

[5]. Kelwin Fernandes, Jaime S. Cardoso, and Jessica Fernandes. 'Transfer Learning with Partial Observability Applied to Cervical Cancer Screening.' Iberian Conference on Pattern Recognition and Image Analysis. Springer International Publishing, 2017.

[6]. NCCC. Cervical cancer. 2010.

[7]. Rama Praba PS, Ranganathan H. Comparing different classifiers for automatic lesion detection in cervix

[8]. M. Nunez, "Decision Tree Induction Using Domain Knowledge" in Current Trends in Knowledge Acquisition, Amsterdam:IOS Press, 1990.

[9]. J. Kern, G. Dezelic, M. TezakBencic, T. Durrigl, "Medical Decision Making Using Inductive Learning Program", Proceedings of 1st Congress on Yougoslav Medical Informatics, pp. 221-228, Dec 6-8, 1990.

[10]. "Fact sheet No. 297: Cancer", World Health Organization, 012007.

\section{Cite this article as :}

Tenali Pranuthi , "Predicting Cervical Cancer Cases Resulting in Biopsies Using Machine Learning Techniques ", International Journal of Scientific Research in Computer Science, Engineering and Information Technology (IJSRCSEIT), ISSN : 24563307, Volume 7 Issue 6, pp. 28-37, NovemberDecember 2021.

Journal URL : https://ijsrcseit.com/CSEIT217616 